\title{
A discrete anatomy of the neutrino mass matrix
}

\author{
G.K. Leontaris ${ }^{a}$ and N.D. Vlachos ${ }^{b}$ \\ ${ }^{a}$ Theoretical Physics Division, Ioannina University, GR-45110 Ioannina, Greece \\ ${ }^{b}$ Theoretical Physics Division, Aristotle University, GR-54124 Thessaloniki, Greece
}

\begin{abstract}
We investigate the possibility of expressing the charged leptons and neutrino mass matrices as linear combinations of generators of a single finite group. Constraints imposed on the resulting mixing matrix by current data restrict the group types, but allow a non zero value for the $\theta_{13}$ mixing angle.
\end{abstract}




\section{On the discrete origin of the neutrino mass}

It is well known that neutrino oscillations are tightly connected to the existence of non-zero neutrino masses and the mixing in the leptonic sector. Recent neutrino data are in accordance with two large mixing angles and a tiny value for the third one. More precisely, adopting the parametrization

$$
C=\left(\begin{array}{lll}
c_{12} c_{13} & c_{13} s_{12} & s_{13} \\
-c_{23} s_{12}-c_{12} s_{13} s_{23} & c_{12} c_{23}-s_{12} s_{13} s_{23} & c_{13} s_{23} \\
s_{12} s_{23}-c_{12} c_{23} s_{13} & -c_{23} s_{12} s_{13}-c_{12} s_{23} & c_{13} c_{23}
\end{array}\right)
$$

(where $c_{i j} \equiv \cos \theta_{i j}$ etc) for the leptonic mixing matrix, the $3 \sigma$ range of the angles is given by

$$
\begin{aligned}
\sin ^{2} \theta_{12} & =[0.26-0.36] \\
\sin ^{2} \theta_{23} & =[0.34-0.66] \\
\sin ^{2} \theta_{13} & =[0.017-0.031] .
\end{aligned}
$$

It has been suggested [1]-21] that the given structure of the mixing matrix indicates the existence of underlying symmetries. Such ideas stimulated the interest on Yukawa superpotentials invariant under discrete groups such as the permutation groups $S_{4}, A_{4}$ etc. The simplest implementation of such groups into the lepton sector has predicted a tri-bimaximal (TB) mixing [1] with a strictly zero value for $\theta_{13}$. However, recent experimental findings suggest a small non-zero value around $\theta_{13} \approx 9^{0}$. In fact, it has been shown that it is possible to generate a non-zero $\theta_{13}$ value even in the context of the minimal TB mixing scenario by introducing in the neutrino texture of [1] an appropriate phase [11, 12], whose acceptable values lie within the $\theta_{13}$ experimental range. In the present work we wish to investigate other possibilities which involve more elaborate forms of mass textures. The motivation for such an analysis is that effective models emerging from unified theories in higher dimensions involve a variety of continuous or discrete groups which act as family symmetries. Although these usually imply mass matrices which look complicated compared to the one above (see for example [22]), yet they are subject to constraints imposed by the underlying symmetries.

\section{Formulation}

It has been shown that small permutation groups like $S_{4}, A_{4}$ constitute good approximate symmetries which, under specific alignments of the vacuum expectation values of the various Higgs fields, generate $m_{\ell, \nu}$ matrices compatible with TB-mixing. Many other finite groups have also been explored in detail [8]-[10].

One way for obtaining a non-zero value for $\theta_{13}$ is to consider deformations of the simple TB mass matrices. Then, we can explore the possibility of still having an underlying group structure in this new landscape. However, since the $\theta_{13}$ value differs substantially from the values of the other two angles, the mixing matrix becomes more involved, hence it is not obvious whether such a finite symmetry exists. Consequently, it is rather difficult to tackle this problem in a straightforward 
manner. Therefore, we will follow a different path and choose to expand the mass matrices in terms of appropriate representations of finite group elements.

\subsection{Expanding mass matrices in terms of discrete group elements.}

One of the interesting properties of the simple TB mass matrix structure is that the diagonalising matrix is independent of the mass eigenvalues. In a previous work, having this in mind and using the Cayley Hamilton theorem [12], we proposed to write a $3 \times 3$ Hermitean mass matrix $M$ in the form

$$
M=c_{1} I+c_{2} U+c_{3} U^{2}
$$

where $U$ is a $3 \times 3$ unitary matrix. Without loss of generality we imposed the condition $\operatorname{det} U=1$, so that $U$ can always be brought in the form

$$
D=\left(\begin{array}{ccc}
e^{i \lambda} & 0 & 0 \\
0 & 1 & 0 \\
0 & 0 & e^{-i \lambda}
\end{array}\right)
$$

by means of a similarity transformation (modulo permutations of the eigenvalues). Observing that $U$ and $M$ can be simultaneously diagonalised, it follows that the mixing associated to the mass matrix $M$ can be simply obtained by diagonalising the matrix $U$. It is then straightforward to see that the eigenmasses are given only in terms of the coefficients $c_{i}$, and the value of the phase $\lambda$,

$$
\begin{aligned}
m_{1}-m_{3} & =2 i\left(c_{2} \sin \lambda+c_{3} \sin 2 \lambda\right) \\
m_{1}+m_{3} & =2\left(c_{1}+c_{2} \cos \lambda+c_{3} \cos 2 \lambda\right) \\
m_{2} & =c_{1}+c_{1}+c_{2} .
\end{aligned}
$$

Hence, the mass eigenvalues problem is essentially disentangled from the diagonalising matrix.

\section{Deforming the TB-mixing matrix}

Now, suppose that the generators $U$ of the mass matrices form elements of a discrete group. It follows that they must satisfy relations of the form $U^{n}=1$ for some integer value of $n$. Their eigenvalues will be $e^{\frac{2 \pi i}{n}}, e^{-\frac{2 \pi i}{n}}, 1$ in some order and can be diagonalised by means of a unitary transformation to produce a diagonal matrix $D_{i, n}$ where the subscript $i$ refers to the eigenvalues ordering. This way, for the leptons we have

$$
U_{l}=V_{l} D_{i, n} V_{l}^{\dagger}
$$

and for he neutrinos

$$
U_{\nu}=V_{\nu} D_{j, m} V_{\nu}^{\dagger}
$$

Hence, the mixing matrix is

$$
C=V_{l}^{\dagger} V_{\nu}
$$


If $U_{l}$ and $U_{\nu}$ belong to the same group they must satisfy a relation of the form

$$
\left(U_{l} U_{\nu}\right)^{p}=1
$$

Also,

$$
U_{l} U_{\nu}=V_{l} D_{n} V_{l}^{\dagger} V_{\nu} D_{m} V_{\nu}^{\dagger}=V_{l} D_{n} C D_{m} C^{\dagger} V_{l}^{\dagger}=V_{l} \mathcal{T} V_{l}^{\dagger}
$$

where

$$
\mathcal{T}=D_{n} C D_{m} C^{\dagger}
$$

This way,

$$
\left(U_{l} U_{\nu}\right)^{p}=V_{l} \mathcal{T}^{p} V_{l}^{\dagger}
$$

so we must have $\mathcal{T}^{p}=1$ for some integer $p$. This implies that one eigenvalue of $\mathcal{T}$ must be 1 while the other two must be $e^{\frac{2 \pi i}{p}}, e^{-\frac{2 \pi i}{p}}$ respectively, since the determinant of $\mathcal{T}$ is 1 . The trace of $\mathcal{T}$ equals $1+2 \cos \frac{2 \pi}{p}$. Therefore, we must seek solutions of the form

$$
1+2 \cos \frac{2 \pi}{p}=\operatorname{Tr} \mathcal{T}
$$

for given values of $m$ and $n$. In the cases where $\operatorname{Tr} \mathcal{T}$ takes complex values there is no solution. Let us now define

$$
\begin{aligned}
D_{1, m} & =\text { Diagonal }\left[1, e^{\frac{2 \pi i}{m}}, e^{-\frac{2 \pi i}{m}}\right] \\
D_{2, m} & =\text { Diagonal }\left[e^{\frac{2 \pi i}{m}}, 1, e^{-\frac{2 \pi i}{m}}\right] \\
D_{3, m} & =\text { Diagonal }\left[e^{\frac{2 \pi i}{m}}, e^{-\frac{2 \pi i}{m}}, 1\right] .
\end{aligned}
$$

Next, as a starting point we take the matrix $C$ to be the TB-mixing matrix. This is readily derived from (1) for $\sin ^{2} \theta_{12}=1 / 3, \sin ^{2} \theta_{23}=1 / 2, \theta_{13}=0$ and choosing the phases appropriately, $C$ can be written as

$$
C=\left(\begin{array}{lll}
\sqrt{\frac{2}{3}} & -\frac{1}{\sqrt{3}} & 0 \\
\frac{1}{\sqrt{6}} & \frac{1}{\sqrt{3}} & -\frac{1}{\sqrt{2}} \\
\frac{1}{\sqrt{6}} & \frac{1}{\sqrt{3}} & \frac{1}{\sqrt{2}}
\end{array}\right)
$$

A subsequent search leads to a limited number of solutions depicted in Table 3 . The $m$ value which refers to the neutrinos turns out always to be 2 .

The lepton mass matrix is then written as

$$
\begin{gathered}
M_{l}=c_{1} I+c_{2} U_{l}+c_{3} U_{l}^{2} \\
U_{l}=V_{l} D_{i, n} V_{l}^{\dagger}
\end{gathered}
$$

and the neutrino mass matrix is written as

$$
\begin{gathered}
M_{\nu}=d_{1} I+d_{2} U_{\nu}+d_{3} U_{\nu}^{2} \\
U_{\nu}=V_{\nu} D_{j, m} V_{\nu}^{\dagger}
\end{gathered}
$$




$\begin{array}{lllll}D_{i, n} & D_{j, m} & n & m & p \\ 1 & 1 & 3 & 2 & 4 \\ 2 & 2 & 3 & 2 & 3 \\ 3 & 3 & 2 & 2 & 4 \\ 1 & 3 & n & 2 & 2 \\ 2 & 3 & 2 & 2 & 4 \\ 3 & 2 & 3 & 2 & 3 \\ 1 & 2 & 3 & 2 & 3\end{array}$

Table 1: Solutions for TB-mixing

In fact, $U_{\nu}^{2}=1$ since the only acceptable solutions are for $m=2$ meaning that the coefficient $d_{3}$ does not exist. This reduction in the degrees of freedom creates a degeneracy in the neutrino mass spectrum. This way, for $D_{1,2}$ we get $m_{2}=m_{3}$ for $D_{2,2}$ we get $m_{1}=m_{3}$ and for $D_{3,2}$ we get $m_{1}=m_{2}$ respectively. Thus, we can either have an exact discrete symmetry with a degenerate neutrino spectrum or a non degenerate spectrum with a broken symmetry. In the latter case one may choose a diagonal neutrino mass matrix i.e. $M_{d}=\operatorname{Diagonal}\left[m_{1}, m_{2}, m_{3}\right]$ and construct the actual matrix through the relation

$$
M_{\nu}=V_{\nu} M_{d} V_{\nu}^{\dagger}
$$

The matrices $V_{l}$ and $V_{\nu}$ are arbitrary unitary matrices connected by the relation

$$
C=V_{l}^{\dagger} V_{\nu}
$$

We now want to generalise the TB mixing in order to accomodate recent data. To that end, let us now assume that the mixing matrix $C$ takes the general form (1).

We may again construct the matrix $\mathcal{T}$ as before, using the new mixing matrix. As previously, we must require that

$$
\operatorname{Im} \operatorname{Tr} \mathcal{T}=0, \operatorname{Re} \operatorname{Tr} \mathcal{T}=1+2 \cos \frac{2 \pi}{p} .
$$

The initial search for viable models was done numerically. No solutions were found for $m \neq 2$. Once a potentially working model is found, it can be elaborated analytically. In what follows, we use the previous classification in terms of the diagonal matrices $D_{i, n}, D_{j, m}$ and the three integers $n, m, p$.

\section{Potentially viable Models}

1. Case $(1,1,3,2, p)$. For this set of integers, $\theta_{12}$ and $\theta_{13}$ are related as follows:

$$
\cos \theta_{12}=-\frac{2}{\sqrt{3}} \frac{\cos \frac{\pi}{p}}{\cos \theta_{13}}
$$


Computing $\theta_{12}$ as a function of $p$, we find that the only acceptable value is $p=3$. In this case, we obtain the following relations:

$$
\begin{aligned}
& \cos \theta_{12}=-\frac{2 \cos \frac{\pi}{3}}{\sqrt{3} \cos \theta_{13}} \equiv-\frac{1}{\sqrt{3} \cos \theta_{13}} \\
& \tan 2 \theta_{23}=\frac{1}{2}\left(\frac{\sin \theta_{13}}{\tan \theta_{12}}-\frac{\tan \theta_{12}}{\sin \theta_{13}}\right) .
\end{aligned}
$$

Plots of the angles $\sin ^{2} \theta_{12}, \sin ^{2} \theta_{12}$ as a function of $\theta_{13}$ are shown in fig 1 . We observe that, although $\theta_{12}$ lies within the acceptable range, this is not so for $\theta_{23}$. Therefore, this model is rejected.

2. Case $(2,2,3,2, p)$

Here, $\theta_{12}$ is given by

$$
\sin ^{2} \theta_{12}=\frac{1}{6 \cos ^{2} \theta_{13}}\left(1-2 \cos \frac{2 \pi}{p}\right) .
$$

The only acceptable value is $p=3$ giving

$$
\sin \theta_{12} \cos \theta_{13}=-\frac{1}{\sqrt{3}}
$$

identical to $(1,1,3,2, p)$. However, the angle $\theta_{23}$ differs

$$
\tan 2 \theta_{23}=-\frac{2 \cot 2 \theta_{13}}{\sqrt{3-\sec ^{2} \theta_{13}}}
$$

Plots for $\sin ^{2} \theta_{12}$ and $\sin ^{2} \theta_{23}$ vs $\theta_{13}$ are given in figure 2. This time, both quantities lie within the acceptable experimental ranges, hence this model is compatible with the data.

3. Case $(3,2,3,2, p)$

The resulting formula for $\theta_{12}$ is identical to the one in $(1,1,3,2, p)$

$$
\sin \theta_{12}=-\frac{2 \cos \frac{\pi}{p}}{\sqrt{3} \cos \theta_{13}} .
$$
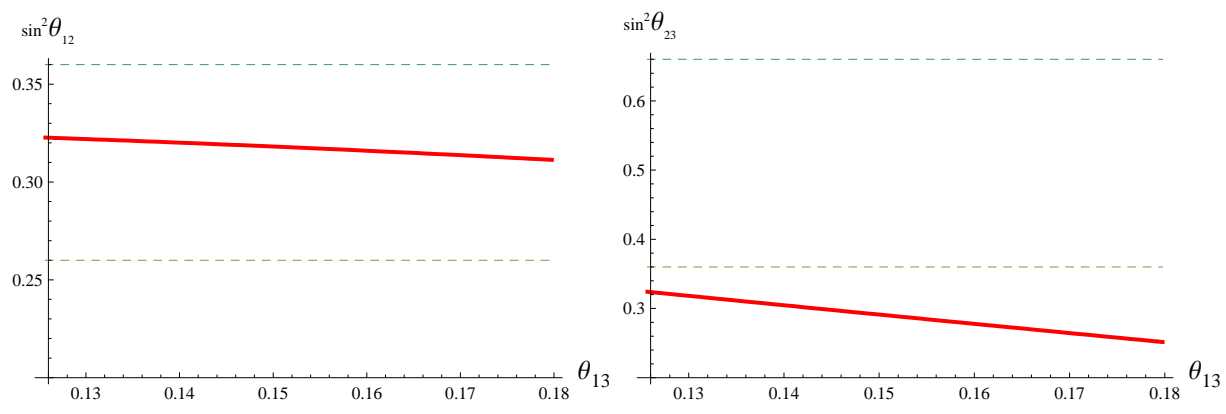

Figure 1: Range for $\sin ^{2} \theta_{12}, \sin ^{2} \theta_{23}$ as a function of $\theta_{13}$ for the model $(2,2,3,3,3)$. We observe that $\sin ^{2} \theta_{23}$ lies outside the $3 \sigma$ range and therefore this model is incompatible with the data. 
Hence, as above, the only acceptable value is $p=3$, leading to the constraint

$$
\sin \theta_{12}=-\frac{1}{\sqrt{3} \cos \theta_{13}}
$$

as in $(1,1,3,2, p)$ and $(2,2,3,2, p)$. For $p=3$ the angle $\theta_{23}$ is given by

$$
\tan 2 \theta_{23}=-\frac{2 \cot 2 \theta_{13}}{\sqrt{3-\sec ^{2} \theta_{13}}}
$$

Comparing with previous cases, we observe that for $p=3$, as far as the mixing is concerned, this model is identical to the second one, therefore it is compatible with data. Setting $\sin \theta_{13}=$ $s$ the mixing matrix becomes

$$
C=\left[\begin{array}{ccc}
\sqrt{\frac{2}{3}-s^{2}} & -\frac{1}{\sqrt{3}} & s \\
\frac{\sqrt{3}}{2} s+\frac{1}{2} \sqrt{\frac{2}{3}-s^{2}} & \frac{1}{\sqrt{3}} & \frac{1}{2} s-\frac{\sqrt{3}}{2} \sqrt{\frac{2}{3}-s^{2}} \\
-\frac{\sqrt{3}}{2} s+\frac{1}{2} \sqrt{\frac{2}{3}-s^{2}} & \frac{1}{\sqrt{3}} & \frac{1}{2} s+\frac{\sqrt{3}}{2} \sqrt{\frac{2}{3}-s^{2}}
\end{array}\right]
$$

Since the only allowed generalization requires that $n=3, m=2, p=3$ we are led to the conclusion that the only symmetry group that can connect the charged lepton and the neutrino mass matrices is the discrete group $A_{4}$. Observe that in the adopted formalism the middle column of $C$ remains unchanged i.e. given by the column vector $\left\{-\frac{1}{\sqrt{3}}, \frac{1}{\sqrt{3}}, \frac{1}{\sqrt{3}}\right\}^{T}$. It turns out that the so constructed generalisation of the TB mixing matrix utilises the freedom of making linear transformations inside the degenerate neutrino subspace to create a non vanishing value for the $\theta_{13}$ angle. This subspace is obviously orthogonal to the $\left\{-\frac{1}{\sqrt{3}}, \frac{1}{\sqrt{3}}, \frac{1}{\sqrt{3}}\right\}$ axis.

As an example, we compute the mixing matrix for $\theta_{13}=\pi / 20$, where the elements although exhibiting significant deviations from the TB case, are still consistent with data.

$$
V=\left[\begin{array}{ccc}
0.801371 & -0.57735 & 0.156434 \\
0.536162 & 0.57735 & -0.61579 \\
0.265209 & 0.57735 & 0.772225
\end{array}\right]
$$
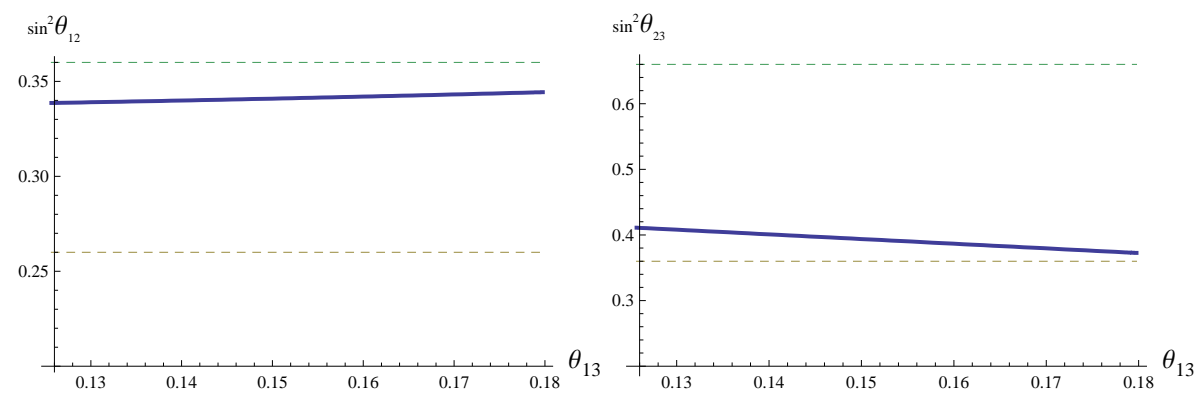

Figure 2: Range for $\theta_{12}, \theta_{23}$ as a function of $\theta_{13}$ for the model $(2,2,3,3,3)$. 


\section{The mass spectrum}

We have found two models with identical predictions with respect to the mixing. The models differ only in the structure of the charged lepton mass matrix implying different coefficients $c_{1,2,3}$ for the two cases. Namely, if the mass matrix eigenvalues are given by $m_{1}, m_{2}, m_{3}$, and we define $\Delta m_{i j}=m_{i}-m_{j}$, the coefficient functions are given by

$$
\begin{aligned}
& c_{1}^{2}=-\frac{1}{8} \csc ^{2} \frac{\pi}{n} \sec \frac{\pi}{n}\left[-2 m_{2} \cos \frac{\pi}{n}+m_{3} e^{\frac{3 i \pi}{n}}+m_{1} e^{-\frac{3 i \pi}{n}}\right] \\
& c_{2}^{2}=\frac{1}{4} \csc ^{2} \frac{\pi}{n}\left[\cos \frac{2 \pi}{n}\left(\Delta m_{12}-\Delta m_{23}\right)-i \sin \frac{2 \pi}{n} \Delta m_{13}\right] \\
& c_{3}^{2}=\frac{1}{8} \csc \frac{\pi}{n}\left[\csc \frac{\pi}{n}\left(\Delta m_{23}-\Delta m_{12}\right)+i \sec \frac{\pi}{n} \Delta m_{13}\right]
\end{aligned}
$$

for $D_{2, n}$ and

$$
\begin{aligned}
c_{1}^{3} & =-\frac{1}{8} \csc ^{2} \frac{\pi}{n} \sec \frac{\pi}{n}\left[-2 m_{3} \cos \frac{\pi}{n}+m_{2} e^{\frac{3 i \pi}{n}}+m_{1} e^{-\frac{3 i \pi}{n}}\right] \\
c_{2}^{3} & =\frac{1}{4} \csc ^{2} \frac{\pi}{n}\left[\cos \frac{2 \pi}{n}\left(\Delta m_{13}+\Delta m_{23}\right)-i \sin \frac{2 \pi}{n} \Delta m_{12}\right] \\
c_{3}^{3} & =\frac{1}{8} \csc \frac{\pi}{n}\left[-\csc \frac{\pi}{n}\left(\Delta m_{13}+\Delta m_{23}\right)+i \sec \frac{\pi}{n} \Delta m_{12}\right] .
\end{aligned}
$$

for $D_{3, n}$.

The results found show that for leptons $n=3$ while for neutrinos we get $m=2$. The corresponding coefficient functions are:

- Leptons

$$
\begin{aligned}
c_{1}^{2} & =\frac{1}{3}\left(m_{1}+m_{2}+m_{3}\right) \\
c_{2}^{2} & =\frac{1}{6}\left(2 m_{2}-m_{1}-m_{3}\right)-\frac{i}{2 \sqrt{3}}\left(m_{1}-m_{3}\right) \\
c_{3}^{2} & =\frac{1}{6}\left(2 m_{2}-m_{1}-m_{3}\right)+\frac{i}{2 \sqrt{3}}\left(m_{1}-m_{3}\right)
\end{aligned}
$$

and

$$
\begin{aligned}
c_{1}^{3} & =\frac{1}{3}\left(m_{1}+m_{2}+m_{3}\right) \\
c_{2}^{3} & =\frac{1}{6}\left(2 m_{3}-m_{1}-m_{2}\right)-\frac{i}{2 \sqrt{3}}\left(m_{1}-m_{2}\right) \\
c_{3}^{3} & =\frac{1}{6}\left(2 m_{3}-m_{1}-m_{2}\right)+\frac{i}{2 \sqrt{3}}\left(m_{1}-m_{2}\right)
\end{aligned}
$$

- Neutrinos

For the neutrinos $m=2$. The $D_{2}$ and the corresponding neutrino matrices are given by:

$$
\begin{aligned}
D_{2} & =\text { Diagonal }[-1,1,-1] \\
M & =\operatorname{Diagonal}\left[m_{1}, m_{2}, m_{3}\right] .
\end{aligned}
$$


In this case, it is clear that the neutrino mass spectrum turns out to be degenerate since $D_{2}{ }^{2}=1$ implying $m_{1}=m_{3}$. In order to establish a breaking pattern we must write

$$
M=d_{1} I+d_{2} D_{2}+R_{2}
$$

where $R_{2}$ is the remainder term to be determined.

$$
R_{2}=\left[\begin{array}{ccc}
m_{1}-d_{1}+d_{2} & 0 & 0 \\
0 & m_{1}-d_{1}-d_{2} & 0 \\
0 & 0 & m_{3}-d_{1}+d_{2}
\end{array}\right] .
$$

Each non vanishing element must be proportional to the same mass difference in order to have a breaking pattern. Since

$$
\left(R_{1}\right)_{11}-\left(R_{1}\right)_{33}=m_{1}-m_{3}
$$

this mass difference is $m_{1}-m_{3}$ if no special relations between neutrino masses are assumed. So we have

$$
\begin{aligned}
& m_{1}-d_{1}+d_{2}=r_{1}\left(m_{1}-m_{3}\right) \\
& m_{2}-d_{1}-d_{2}=r_{2}\left(m_{1}-m_{3}\right) \\
& m_{3}-d_{1}+d_{2}=r_{3}\left(m_{1}-m_{3}\right)
\end{aligned}
$$

with $r_{1}-r_{3}=1$. Solving for $d_{1}, d_{2}$ we get

$$
\begin{aligned}
& d_{1}=\frac{1}{2}\left(m_{1}+m_{2}\right)-\frac{1}{2}\left(r_{1}+r_{2}\right)\left(m_{1}-m_{3}\right) \\
& d_{2}=-\frac{1}{2}\left(m_{1}-m_{2}\right)+\frac{1}{2}\left(r_{1}-r_{2}\right)\left(m_{1}-m_{3}\right)
\end{aligned}
$$

for arbitrary $r_{1}$ and $r_{2}$. A different breaking pattern would require invariant relations between the neutrino masses. For instance if we require that

$$
\begin{aligned}
& m_{1}-d_{1}+d_{2}=r_{1}\left(m_{1}-m_{2}\right) \\
& m_{2}-d_{1}-d_{2}=r_{2}\left(m_{1}-m_{2}\right) \\
& m_{3}-d_{1}+d_{2}=r_{3}\left(m_{1}-m_{2}\right)
\end{aligned}
$$

consistency implies that

$$
r_{3}=r_{1}+\frac{m_{3}-m_{1}}{m_{1}-m_{2}}
$$

independent of the masses i.e. $\frac{m_{3}-m_{1}}{m_{1}-m_{2}}=\mu$, and

$$
\begin{gathered}
d_{1}=\frac{1}{2}\left(m_{1}+m_{2}\right)-\frac{1}{2}\left(r_{1}+r_{2}\right)\left(m_{1}-m_{2}\right) \\
d_{2}=\frac{1}{2}\left(r_{1}-r_{2}-1\right)\left(m_{1}-m_{2}\right) .
\end{gathered}
$$

Finally, we end up our analysis by presenting the first order corrections to the charged leptons mass matrix written as an expansion in terms of $s=\sin \theta_{13}$.

$$
m_{\ell} \approx A+s B
$$


where

$$
A=\left(\begin{array}{ccc}
\frac{1}{6}\left(4 m_{1}+m_{2}+m_{3}\right) & \frac{-2 m_{1}+m_{2}+m_{3}}{3 \sqrt{2}} & \frac{m_{3}-m_{2}}{2 \sqrt{3}} \\
\frac{-2 m_{1}+m_{2}+m_{3}}{3 \sqrt{2}} & \frac{1}{3}\left(m_{1}+m_{2}+m_{3}\right) & \frac{m_{3}-m_{2}}{\sqrt{6}} \\
\frac{m_{3}-m_{2}}{2 \sqrt{3}} & \frac{m_{3}-m_{2}}{\sqrt{6}} & \frac{m_{2}+m_{3}}{2}
\end{array}\right)
$$

and

$$
B=\left(\begin{array}{ccc}
\frac{\left(m_{2}-m_{3}\right)}{\sqrt{2}} & \frac{1}{2}\left(m_{2}-m_{3}\right) & \frac{\left(2 m_{1}-m_{2}-m_{3}\right)}{\sqrt{6}} \\
\frac{1}{2}\left(m_{2}-m_{3}\right) & 0 & \frac{\left(-2 m_{1}+m_{2}+m_{3}\right)}{2 \sqrt{3}} \\
\frac{\left(2 m_{1}-m_{2}-m_{3}\right)}{\sqrt{6}} & \frac{\left(-2 m_{1}+m_{2}+m_{3}\right)}{2 \sqrt{3}} & \frac{\left(m_{3}-m_{2}\right)}{\sqrt{2}}
\end{array}\right)
$$

\section{Conclusions}

In this letter we have examined the structure of the lepton and neutrino mass matrices assuming that they can be expanded in a basis of finite group generators. This procedure has been proven useful for putting some order on the enormous number of possibilities given in the literature in a systematic and mathematically consistent way. Various models are classified by means of three integer numbers which define the group. The calculations show that only the $A_{4}$ group can reproduce current data by allowing a non zero value for the $\theta_{13}$ mixing angle. Exact group symmetry introduces a degeneracy in the neutrino spectrum which has to lifted by means of an external breaking mechanism.

Aknowledgments. The authors would like to thank the CERN theory group for its kind hospitality while this work was in preparation. The research Project is co-financed by the European Union - European Social Fund (ESF) and National Sources, in the framework of the program "THALIS" of the "Operational Program Education and Lifelong Learning" of the National Strategic Reference Framework (NSRF) 2007-2013. 


\section{References}

[1] P. F. Harrison, D. H. Perkins and W. G. Scott, "Tri-bimaximal mixing and the neutrino oscillation data," Phys. Lett. B 530 (2002) 167 hep-ph/0202074.

[2] E. Ma, "A(4) symmetry and neutrinos with very different masses," Phys. Rev. D 70 (2004) 031901 hep-ph/0404199].

[3] G. Altarelli and F. Feruglio, "Tri-bimaximal neutrino mixing, A(4) and the modular symmetry," Nucl. Phys. B 741 (2006) 215 hep-ph/0512103.

[4] C. S. Lam, "Symmetry of Lepton Mixing," Phys. Lett. B 656 (2007) 193 arXiv:0708.3665 [hep-ph]].

[5] G. Altarelli, F. Feruglio and L. Merlo, "Revisiting Bimaximal Neutrino Mixing in a Model with S(4) Discrete Symmetry,” JHEP 0905 (2009) 020 arXiv:0903.1940 [hep-ph]].

[6] I. de Medeiros Varzielas, G. G. Ross and M. Serna, "Quasi-degenerate neutrinos and tri-bimaximal mixing," Phys. Rev. D 80 (2009) 073002 arXiv:0811.2226 [hep-ph]].

[7] R. N. Mohapatra, S. Antusch, K. S. Babu, G. Barenboim, M. -C. Chen, A. de Gouvea, P. de Holanda and B. Dutta et al., Rept. Prog. Phys. 70 (2007) 1757 hep-ph/0510213.

[8] H. Ishimori, T. Kobayashi, H. Ohki, Y. Shimizu, H. Okada and M. Tanimoto, "NonAbelian Discrete Symmetries in Particle Physics," Prog. Theor. Phys. Suppl. 183 (2010) 1 arXiv:1003.3552 [hep-th]].

[9] G. Altarelli and F. Feruglio, "Discrete Flavor Symmetries and Models of Neutrino Mixing," Rev. Mod. Phys. 82 (2010) 2701 arXiv:1002.0211 [hep-ph]].

[10] S. F. King and C. Luhn, "Neutrino Mass and Mixing with Discrete Symmetry," arXiv:1301.1340 [hep-ph].

[11] Z. -z. Xing, "Nearly tri bimaximal neutrino mixing and CP violation," Phys. Lett. B 533 (2002) 85 hep-ph/0204049.

[12] G. K. Leontaris and N. D. Vlachos, "Knitting neutrino mass textures with or without Tri-Bi maximal mixing," Phys. Lett. B 702 (2011) 34 arXiv:1103.6178 [hep-ph]].

[13] D. A. Dicus, S. -F. Ge and W. W. Repko, "Neutrino mixing with broken $S_{3}$ symmetry," Phys. Rev. D 82 (2010) 033005 arXiv:1004.3266 [hep-ph]].

[14] X. -G. He and A. Zee, "Minimal Modification to Tri-bimaximal Mixing," Phys. Rev. D 84 (2011) 053004 arXiv:1106.4359 [hep-ph]].

[15] S. F. King and C. Luhn, "Trimaximal neutrino mixing from vacuum alignment in A4 and S4 models," JHEP 1109 (2011) 042 arXiv:1107.5332 [hep-ph]]. 
[16] D. Meloni, "Bimaximal mixing and large theta13 in a SUSY SU(5) model based on S4," JHEP 1110 (2011) 010 arXiv:1107.0221 [hep-ph]].

[17] C. Luhn, S. Nasri and P. Ramond, "Tri-bimaximal neutrino mixing and the family symmetry semidirect product of Z(7) and Z(3)," Phys. Lett. B 652 (2007) 27 [arXiv:0706.2341 [hep-ph]].

[18] D. A. Dicus, S. -F. Ge and W. W. Repko, "Neutrino mixing with broken $S_{3}$ symmetry," Phys. Rev. D 82 (2010) 033005 arXiv:1004.3266 [hep-ph]].

[19] P. S. Bhupal Dev, R. N. Mohapatra and M. Severson, "Neutrino Mixings in SO(10) with Type II Seesaw and $\theta_{13}, "$ Phys. Rev. D 84 (2011) 053005 [arXiv:1107.2378 [hep-ph]].

[20] D. Hernandez and A. Y. .Smirnov, "Lepton mixing and discrete symmetries," Phys. Rev. D 86 (2012) 053014 arXiv:1204.0445 [hep-ph]].

[21] M. Cannoni, J. Ellis, M. E. Gomez and S. Lola, "Neutrino textures and charged lepton flavour violation in light of theta13, MEG and LHC data," arXiv:1301.6002 [hep-ph].

[22] J. R. Ellis, G. K. Leontaris, S. Lola and D. V. Nanopoulos, Eur. Phys. J. C 9 (1999) 389 hep-ph/9808251. 